\title{
Modelling impact of advertising and optimizing advertising policy: an application in recreation
}

\author{
Berend WIERENGA \\ Depariment of Marketing and Marketing Research. Wageningen \\ Agricultural University, 6706 KN Wageningen, Netherlands
}

Received January 1980

Revised December 1980

This paper deals with the problem of the desirable level of advertising expenditure, the optimal distribution of this expenditure in time and the allocation over the media: TV, radio and newspaper for a recreation park in the Netherlands.

Although the model is developed for the specific situation of this park, in principle it can be applied in all situations where the interest is in short-term (day-by-day) effects of promotional activities on sales. Examples are: other situations in the recreation and leisure business, cultural events (theatre, cinema) and sales promotions (e.g. weekend offerings) for products in supermarkets.

First a model was specified and estimated that relates number of visitors to advertising effort. It also takes into account non-advertising variables that affect the number of visitors.

Then this model was used in a heuristic advertising planning procedure, which by means of incremental analysis, for a given budget level searches for the optimal allocation of the advertising budget over media and time.

With this procedure, ways to readjust the advertising policy were found: by allocating the budget differently over media and time and by changing the overall budget level.

Several recommendations were made to the management of the park, a number of which have already been implemented.

\section{Introduction}

This study describes a model building approach to the problem of determining the optimal level of

The author wishes to thank Jaap Bijkerk, Gerard Verwey and Bart Braam for their help in carrying out the computations. Frans de Hey did much exploratory work for this study. Very useful comments on an earlier version of this paper were obtained from Paul van Beek (Wageningen Agricultural University, Dept. of Mathematics), Peter Leeflang (University of Groningen) and an anonymous EJOR-referee.

North-Holland Publishing Company

European Journal of Operational Research 8 (1981) 235-2.48 the advertising budget and of allocating this budget over media and over time in the situation of a recreation park.

Up to now most of the model building for marketing decision making has been done in the area of frequently bought consumer goods. Entertainment and recreation activities have received less attention, although some work can be mentioned: a study in planning performing arts series by Weinberg and Shachmut [9] and a volume of articles edited by Ladany [2] with applications such as outdoor recreation parks, camping, tourism and a zoological garden. So the research described in this paper refers to a relatively new area of application.

There is another feature that makes the research repurted here different from most of the work on the empirical measurement of advertising effects. The approach commonly followed is that advertising expenditures referring to particular periods (for example monthly or yearly data may be used) are statistically related to sales in the same periods. For a recent overview of this type of work, see Little [4].

However, in this paper the emphasis is on measuring the effects of advertisements on a day-by-day basis. The reason is that there are tremendous differences in sales potential among the various days of the week. For the efficient planning of advertising it is important to know that, for example, a tv-spot placed on Friday may generate a very different number of additional sales (visitors in this application) than the same spot if placed on Thursday. Therefore this paper develops a model for advertising effects on a day-by-day basis.

The application of this model is not limited to this particular application of a recreation park. It can be used in all situations where the interest is in short-term (day-b $b_{j}$-day) efiects of promotional activities. Examples are: other situations in the recreation and leisure business, cultural events (theatre, cinema) and sales promotions (e.g. special weekend offerings) for products in supermarkets. 
The questions asked by the management of the recreation park are very typical for the type of questions one would generally have with respect to advertising activities and the framework adopted to answer these questions can readily be transferred to other contexts.

The recreation park, located somewhere in the Netherlands, uses three types of advertising to attract visitors: television spots, radio spots and print ads in daily newspapers. The management of the park has the following questions about its advertising policy.

- Is the current level of total advertising expenditures right?

- How should the advertising budget be allocated over the three media?

- When, i.e. on which days during the 8 months of the year when the park is open, should the advertisements be placed?

This paper tries to answer these three questions by developing a model for the relationship between advertising effort and daily numbers of visitors. This model is the basic element of a heuristic procedure for the allocation of advertising expenditures over the three media and over the year. It can also be used to evaluate the consequences of alternative levels of the advertising budget.

The remaining part of the paper is organized as follows.

Firstly the situation is described briefly. In the next section the model for the daily number of visitors is developed and the parametrization of this model, using historical data is described. Also the results of a validation analysis are given. Subsequently the heuristic advartising planning procedure is dealt with and the results obtained with this procedure are presented. Then follow the conclusions, recommendations and implementation issues. A summary concludes the paper.

\section{Brief description of the situation}

The park was opened in 1969 and currently receives 600000 to 700000 visitors a year. It spends about $10 \%$ of total turnover on advertising, newspapers receiving the largest share (53\%), followed by TV $(39 \%)$ and radio (8\%). The management feels that advertising is essential for the attraction of visitors. This feeling is $\mathrm{artly}$ based on the lesson learnt in 1972 when TV and radio advertis- ing were dropped and newspaper advertising was drastically reduced. As a result the number of

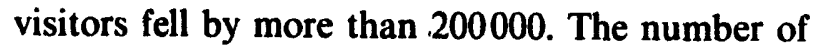
visitors recovered in the following years when advertising was resumed.

The park is open from March 1 to October 31, every day of the week.

According to the number of visitors three periods can be distinguished:

(1) spring/autumn season: the months March, April, May, September and October;

(2) summer season: the months June, July and August, excluding the vacation peak;

(3) vacation peak: the three-week period in which the workers in the major Netherlands industries, construction, metal and related industries, have their holidays. The time of this vacation varies from year to year, it falls for the greater part or completely within July.

The average number of daily visitors in the summer season is 4 times as high as in the spring/autumn season, in the vacation peak it is 9 times as high. The management of the park distinguishes between two types of visitor: individuals (including small groups such as families) and visiting groups. The latter are school classes, groups of aged persons, etc. Our analysis in the following sections only refers to the first group: the individual visitors, who represent about $80 \%$ of the total number of visitors.

This is because the advertising activities analysed here are specially directed towards these 'individual visiters'. For the other part of the market: school classes, etc., there are special promotional programs, such as direct mail, visits by sales people, etc., which are outside the scope of this analysis.

At the time of the main analysis data were available on the daily number of visitors for the ye? rs 1970 to 1977 and, also from 1970 onwards, the emission dates of TV and radio spots and the publishing dates of the printed advertisements. Afterwards also the data for 1978 and 1979 became available. These data were used in the validation study reported later on. For the newspaper ads each publishing date represents the placement of an advertisement in 15 Netherlands daily newspapers (national as well as regional) on the same day. The content and layout of the advertisements in all three media did not vary much over the years.

Marketing variables other than advertising do 
not seem very important for explaining the variation in numbers of visitors. The mix element product, the park itself, did not undergo major changes over the years; the mix element admission price roughly followed the inflation.

\section{The model}

\subsection{Factors to be included and structure of the model}

The purpose was to develop a model representing the effects of the various advertising activities on the daily number of visitors. However, when doing this, one has to take into account factors other than advertising that also influence the number of visitors on a given day. These factors are:

- day of the week (D). There is a certain pattern in the distribution of visitors over the week. Especially weekend days are differenc from week days;

- month (M). Because of the large differences in the numbers of visitors three different seasons have already been distinguished. However, even within each season there are clear differences from month to month;

- weather $(\mathrm{W})$;

- schoolvacations (SV);

- special holidays (SH): Easter, Ascension Day, Whitsun, the Queen's Birthday, Liberation Day;

- year (Y).

It was assumed that together with advertising (ADV) : hese factors are the major variables that influence the daily number of visitors (V). Obviously there are interactions between these factors. For example the difference between the number of visitors on a weekend day and a week day (i.e. the weekend effect) is higher in a month with a high general level of daily visitors than in a month with a low level of daily visitors.

To give another example: an advertisement will have a greater effect on the number of visitors when placed just before a major holiday than before an ordinary week day. Because of these interactions a multiplicative model was specified with a basic structure similar to that of Little's BRANDAID-model, Little [3]. Our model for the daily number of visitors has the following form:

$$
\begin{aligned}
\mathrm{V}_{t}= & c \cdot f_{\mathrm{D}, t} \cdot f_{\mathrm{M}, t} \cdot f_{\mathrm{W}, t} \cdot f_{\mathrm{SV}, t} \cdot f_{\mathrm{SH}, t} \\
& \cdot f_{\mathrm{Y}, t} \cdot f_{\mathrm{ADV}, t},
\end{aligned}
$$

where $V_{t}$ is the number of visitors on day $t, c$ a constant and the factors $f$ represent multiplicative factors corresponding with the variables just mentioned. To explain the nature of these multiplicative factors we take $f_{\mathrm{D}, \ell}$, the day-of-the-week factor as an example. There is a reference situation in which $f_{\mathrm{D}, t}$ takes the value 1 . Here we arbitrarily chose Monday for the reference situation. For the other days of the week $f_{\mathrm{D}, t}$ represents the ratio of the number of visitors on that day to the number of visitors on Monday (all other circumstances being equal). The other non-advertising factors (the advertising effect is dealt with in the next subsection) should be interpreted in a similar way.

With respect to the year effects there is a steady decline in the annual number of visitors, probably because of the decreasing 'newness' of the park (at the start there are many people who want to see the park at least once) and because of the decreasing birth rate (the park is especially attractive to small children). In the ultimate advertising planning model the year effect is modelled by a negative growth curve. In the estimation phase the values of $f_{Y}$ for the various years are estimated directly, taking 1974 as the reference situation.

\subsection{The advertising submodel}

The advertising effect, represented by the factor $f_{\mathrm{ADV}, t}$ in the visitors function (1) consists of advertising by three different media: television (TV), radio (RA) and daily newspaper (DN). Within the advertising submodel we assume a multiplicative structure as:

$f_{\mathrm{ADV}, t}=\prod_{j=1}^{3} \mathrm{fa}_{j t}$,

where $\mathrm{fa}_{j l}$ is the advertising factor for medium $j \mathrm{o}$ day $t$ and $j=1,2,3$ corresponds with television, radio and newspaper respectively.

So specified the advertising subm $\lrcorner$ del has the same (multiplicative) structure as the main model, which makes estimation easy (Section 3.3). Of course this multiplicative assu nption implies tha' advertisements in the three media have a mutually reinforcing effect. Whether or not this is a realistic assumption is ultimately determined by the fit of the moitel to the data.

Nex: the relationship between the advertising factors $\mathrm{fa}_{j t}$ and the past advertising effort for mediun $j$ has to be specified. We assume that this 
relationship has the same form for all three media. Generally, the number of visitors on a given day is not only affected by the most recent advertisement, but there is also a carry-over effect from previous advertising activities, especially as we are working with a model for the daily number of visitors. For example, somebody may see an advertisement of the park on Tuesday, but his first opportunity to visit the park may be in the next weekend or in his children's school vacation. To describe these lagged effects of past advertising a model was developed in which $i_{i}$ is assumed that the effect of an advertisement decreases wits the number of days elapsed since the appearance of that advertisement, according to the hyperbolic function:

$y=1 / x$.

Here

$y=$ index of effect,

$x=$ the number of days elapsed since the advertisement appeared.

The effect of an advertisement at day $t_{0}$ on the number of visitors at day $t\left(t_{0}<t\right)$ is according to (3):

$1 /\left(t-t_{0}\right)$.

This hyperbolic decay function is very straightforward but works very well in this case. Other advertising decay functions have been proposed in the literature, e.g. a proportional decay function, Little and Lodish [5] and an exponential decay function, Vidale and Wolfe [8].

Fig. 1 depicts the decreasing effect of an advertisement in time according to this hyperbolic function. The recreation park is well-known in the Netherlands. The primary task of advertising is to act as a reminder and in this way to trigger visits. Therefore it may be expected that the greater part of the advertising effect occurs rather quickly af ter the placing of the advertisements, as is implied by Fig. 1.

At least in principle, all advertisements that appeared before $t$ have an impact on the number of visitors at $t$. The total cumulative effect, as expressed for day $t$, of all advertisements in medium $j$ that appeared before $t$ is represented by:

$$
\mathrm{um}_{j t}=\sum_{\substack{i \\ t(i)<t}} 1 /\left(t-t_{0}^{j}(i)\right),
$$

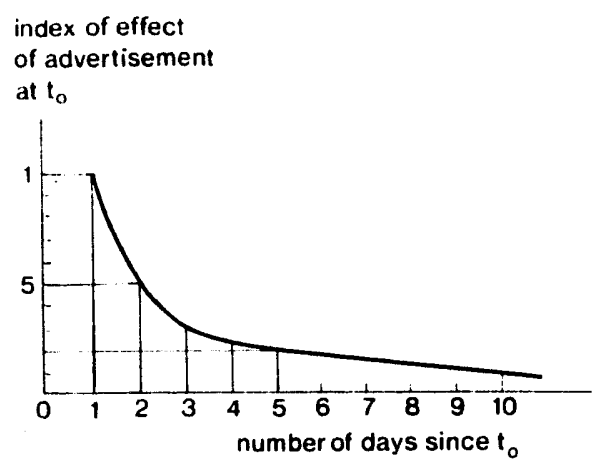

Fig. 1. Hyperbolic decrease in the effect of ar'vertising.

where the index $i$ runs over the consscutive advertisements in medium $j$ and $t_{0}^{j}(1), t_{0}^{j}(2), \ldots$, etc. are the days on which these consecutive advertisements in medium $j$ have appsared.

This way of computing past advertising effects is illustrated in Fig. 2. From this figure it is also clear that the effect of an advertisement is assumed to start the day after it appeared. Usually people plan a visit to the park at least the evening before. Thus the denominator in the hyperbolic function (3) is always greater than or equal to one.

The cumulative advertising effect for a medium, as given by (4), may be considered as the total 'stock of goodwill' caused by advertising in the past.

The factors cum $_{j t}$ could be entered directly into the advertising submodel, i.e. by putting $\mathrm{fa}_{j t}=\operatorname{cum}_{j t}$ for $j=1,2,3, t=1,2, \ldots$

However, such a model would imply that after a long period of no advertising, visits to the park would stop completely. This is not realistic and to make the model more 'robust' we add the constant one to the factor cum $_{j t}$. Furthermore, an exponent $\alpha_{j}$ is introduced to express any differences in ef-

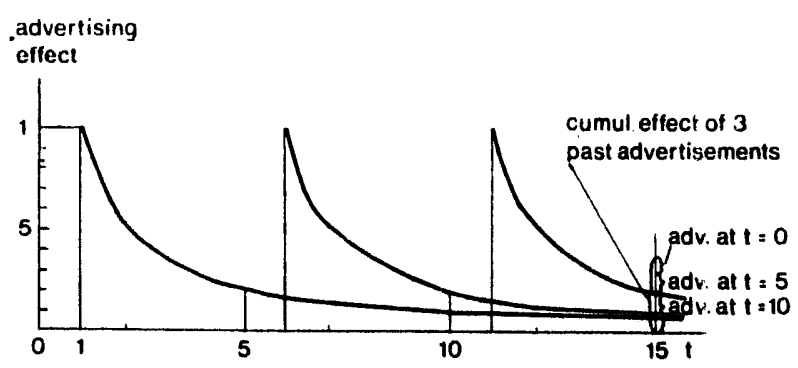

Fig. 2. Illustration of the computation of cumulative advertising effect at $t=15$, caused by advertisements at $t=0, t=5$ and $t=10$ respectively (hyperbolic model). 
fectiveness between the media. In this way we obtain:

$$
\mathrm{fa}_{j t}=\left(1+\operatorname{cum}_{j t}\right)^{\alpha_{j}} \quad(j=1,2,3)
$$

and the complete advertising submodel is:

$$
f_{\mathrm{ADV}, t}=\prod_{j=1}^{3}\left(1+\operatorname{cum}_{j t}\right)^{\alpha_{j}} \text {. }
$$

Note that in this model the advertising factor for a medium becomes one after a long period of no advertisements in this medium. So the latter situation serves as the reference situation here.

At first glance it might be thought that the simple hyperbolic function (3), as depicted in Fig. 1, implies a very rigid assumption about the way the advertising effect decreases over time. However, this is not so in the ultimate model (6), since in this model the advertising effect and its distribution over time are determined by the parameter $\alpha_{j}$, which can be adapted to the particular situation under study.

For $\alpha_{j}<1$, the hyperbolic model implies decreasing incremental effects of additional advertisements in the same medium.

\subsection{The model in a form that can be estimated}

The model is parametrized by least squares regression using historical data for number of visitors, day of the week, weather, advertising activities, etc. All multiplicative factors of (1), except the advertising factor are expressed as powers of $e$. As an example we take $f_{\mathrm{D}, t}$, the factor for the day of the week effect.

We write:

$$
f_{\mathrm{D}, t}=\left(e^{\alpha_{\mathrm{TU}} \mathrm{TU}_{t}}\right)\left(e^{\alpha_{\mathrm{WE}} \mathrm{WE}_{t}}\right) \cdots\left(e^{\alpha_{\mathrm{SU}} \mathrm{SU}_{t}}\right),
$$

where

$$
\begin{aligned}
& \mathrm{TU}_{t}= \begin{cases}1, & \text { if day } t \text { is a Tuesday, } \\
0, & \text { otherwise, }\end{cases} \\
& \mathrm{WE}_{t}= \begin{cases}1, & \text { if day } t \text { is a Wednesday, } \\
0, & \text { if otherwise, }\end{cases}
\end{aligned}
$$

etc.

The parameter $\alpha_{\mathrm{TU}}, \alpha_{\mathrm{WE}}$, etc. represents the effect of Tuesday, Wednesday etc. The other factors: month, weather, etc. are written in an exponential form in a similar way. Now (1) is transformed into:

$$
\begin{array}{r}
V_{t}=\frac{c\left(\mathrm{e}^{\alpha_{\mathrm{TU}}}\right)^{\mathrm{TU}} \cdots\left(e^{\alpha_{\mathrm{SU}}}\right)^{\mathrm{SU}}}{\text { day of the week factors }} \\
\\
\frac{\prod_{j=1}^{3}\left(1+\text { cum }_{j t}\right)^{\alpha,},}{\text { advertising factor }}
\end{array}
$$

other exponential factors corresponding with month, year. weather, school
vacation, ctc.

vacation, ctc.

After logarithmic transformation (7) becomes:

$$
\begin{aligned}
& \ln \mathrm{V}_{t}=\ln c+\underbrace{+\alpha_{\mathrm{TU}} \mathrm{TU}_{t}+\cdots+\alpha_{\mathrm{SU}} \mathrm{SU}_{t}}_{\text {day of the week effect }} \\
& \underbrace{+\ldots+\alpha_{j}}_{\begin{array}{c}
\text { similar terms } \\
\text { for the effects } \\
\text { of month, year, } \\
\text { weather, school } \\
\text { vacation, etc. }
\end{array}}
\end{aligned}
$$

The parameters of (8) can directly be estimated by least squares using information on the number of visitors, the weather, characteristics of the days and advertising activity in the past. The quantities cum $_{j t}$ are straightforwardly computed from knowledge of the appearance dates of past advertisements, using (4).

\section{Estimation and validation results}

\subsection{General}

The parameters were estimated using the data for the period 1970-1976. The data for 1977 were set aside for yalidation purposes. Later on the data from 1978 and 1979 were also used for validation. Weather data referring to the same period were obtained from the Netherlands Weather Bureau'.

Table 1 presents the estimation results. Separate models were estimated for three seasons, as is indicated in Table 1. The models were estimated in logarithmic form, but in Table 1 the coefficients for the non-advertising variables have already been converted into multiplicative factors ${ }^{2}$. Of course $R^{2}$ and the results on the significance of the coefficients refer to the logarithmic model.

I The 'Koninklijk Nederlands Meteorologisch Instituut'.

2 For example in the logarithmic mode for the spring/autumn season the coefficient of the Tuesday variable was 0.203 . The corresponding value in the multiplicative model is $\mathrm{e}^{0.203}=$ 1.26 , which is the number given in Table 1 . 
Table 1

Estimation results (coefficient of non-advertising variables converted to multiplicative factors)

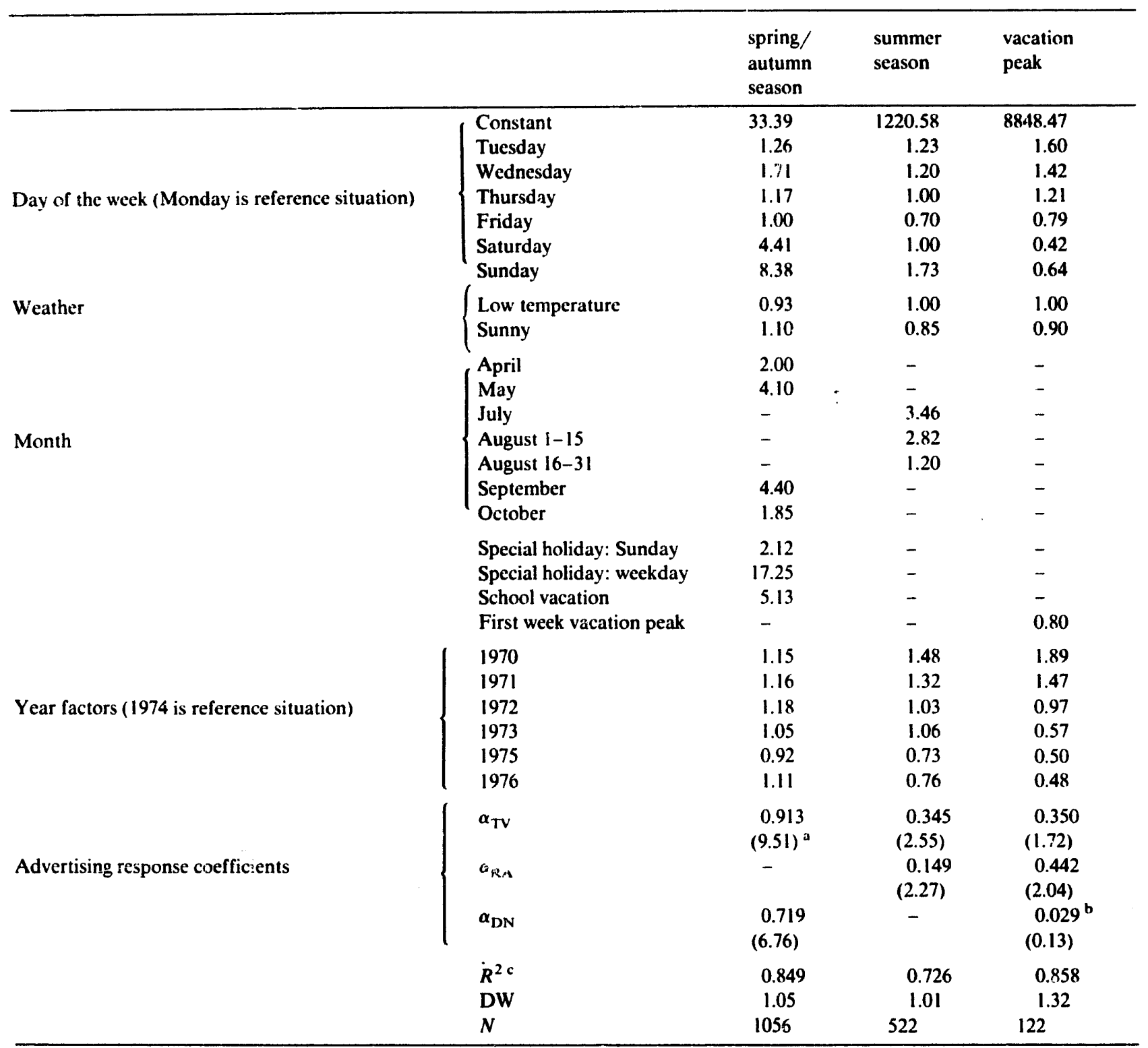

a Numbers in brackets are student- $t$ statistics (for the significance of individual variables).

b Not significant at the $5 \%$ level.

c $R^{2}=$ coefficients of multiple correlation.

$\mathrm{DW}=$ Durbin-Watson statistic (an indication for the presence of autocorrelation).

$N=$ number of observations.

Of the non-advertising factors (except for the year factors) all coefficients in Table 1 that differ from 1.00 refer to coefficients in the logarithmic model that were significant at the $5 \%$ level. Variables not significant at this level were omitted from the model, aiter which the other parameters were re-estimated. Multiplicative factors in Table 1 that have the value 1.00 correspond with such non- significant variables. A dash in Table 1 means that the explanatory variable in question is not applicable for that model.

For the advertising variables in addition to the estimated response coefficients also the $t$-values for these coefficients are reported.

The general conclusion from Table 1 is that the explanation of daily numbers of visitors by the 
model is quiti good. $R^{2}$ ranges from 0.73 for the summer season to 0.86 for the vacation peak. Also the results for the advertising variables seem to be very satisfactory.

Tabie 1 shows evident effects of the day in the week. Moreover this distribution of visitors over the week is very different in different seasons.

The weather variables have some influence. Note that during the summer sunny weather has an opposite effect as compared to spring and autumn. In the summer it decreases, instead of increases the number of visitors.

Table 1 shows that the number of visitors increases towards the summer and decreases towards October. Also within the summer season (with June as the reference month) there are clear differences between the months. Since the vacation peak falls for the greater part or completely in July, no month variables were included in the model for this period.

Special holidays and schoolvacations have an important impact on the number of visitors.

The year factors show a general decline in the number of visitors over the years, which is most notable for the summer season and the vacation peak. The year factors in Table 1 are not strictly decreasing though, because of incidental factors such as the opening of new competing parks and also the oil crisis in 1974 which decreased travelling abroad and thereby increased the visits to the park.

\subsection{Advertising effects}

All advertising response coefficients reported in Table 1 are significant at the 5\% level, except the coefficient for newspapers in the vacation peak. For the summer season the estimated response coefficient for daily newspapers was even slightly negative, so that the newspaper variable was removed from the equation for that season and the other coefficients were re-estimated. Since the park never used radio advertising in the spring/autumn season, no coefficient for radio could be estimated for that season.

There were no high intercorrelations between advertising and non-advertising factors, except a correlation coefficient of -0.78 between the TVvariable and the year factor 1972 in the summer season (1972 was the year without TV-advertising). However, re-estimation of the parameters for the model after removing the data from 1972 practi- cally produced the same regression coefficients.Intercorrelations among advertising variables were small or of modest size (smaller than 0.5 ) with one exception: for the vacation peak the correlation coefficient between $\mathrm{TV}$ and radio advertising was 0.73. So it should be kept in mind that for the vacation the effects of television and radio could not be separated completely.

The Durbin-Watson (D.W.) statistics given in Table 1 indicate autocorrelation in all cases. For this reason the Cochrane-Orcutt procedure, as described by Johnston [1, pp. 262-263] was applied. For all three seasons one iteration was sufficient to bring the D.W.-statistics to a value greater than 1.70. This transformation did not cause great changes in the regression coefficients. In the validation process the estimates before removing autocorrelation gave better predictions.

The results in Table 1 demonstrate evident effects of TV-advertising for all seasons. Also the radio advertising in the summer and vacation peak is effective. However, newspaper advertising is only effective in spring and autumn, perhaps because of different reading habits during the summer months.

The magnitudes of the response coefficients for TV and radio imply a larger relative effect of advertising in the spring/autumn season than in the two other seasons. This is not true for the absolute effects since the daily numbers of visitors are much higher during the summer months.

Also a notable result is the relatively high effectiveness of radio in the vacation peak, perhaps because the radio (car radio, transistors) is used more intensively in this period when a large percentage of the Netherlands population is on the road, on a camping or at other vacation facilities.

\subsection{Validation}

The data for 1977,1978 and 1979 represented 'fresh data' that were used for validation purposes. For these years daily numbers of visitors were predicted with the model and compared with the actual numbers. The same was done for the estimation period. Table 2 gives the prediction results, compared with the actual data.

To be able to make predictions for a yeár after 1976 the year factor (see eq. (1)) for such a year has to be known. This factor was computed as follows. It was assumed that the year factor declines according to the negative growth curve:

$g_{t}=\min +(1-\min ) \mathrm{e}^{-a t}$, 
Table 2

Prediction results for the estimation and the validation period

\begin{tabular}{|c|c|c|c|c|c|c|}
\hline & \multicolumn{2}{|c|}{ Estimation period } & \multicolumn{4}{|c|}{ Validation period } \\
\hline & \multirow{2}{*}{$\frac{970}{O^{a}}$} & \multirow{2}{*}{$\frac{1976}{A}$} & \multicolumn{2}{|l|}{1977} & \multirow{2}{*}{$\begin{array}{l}1978 \\
0\end{array}$} & \multirow{2}{*}{1979} \\
\hline & & & o & $\mathbf{A}$ & & \\
\hline$R$ (predicted, actual) & 0.91 & 0.91 & 0.90 & 0.87 & 0.90 & 0.94 \\
\hline $\begin{array}{l}\text { Average deviation between predicted and } \\
\text { actual numbers of visitors per day }\end{array}$ & 754 & 730 & 641 & 742 & 640 & 584 \\
\hline $\begin{array}{l}\text { The same, expressed as percentage of the } \\
\text { average number of visitors per day }\end{array}$ & $31 \%$ & $30 \%$ & $29 \%$ & $34 \%$ & $32 \%$ & $26 \%$ \\
\hline $\begin{array}{l}\text { Average deviation between predicted and } \\
\text { actial number of visitors per week }\end{array}$ & 3405 & 3523 & 3017 & 4149 & 3255 & 3254 \\
\hline $\begin{array}{l}\text { The same, expressed as percentage of the } \\
\text { average number of visitors per week }\end{array}$ & $20 \%$ & $20 \%$ & $20 \%$ & $28 \%$ & $23 \%$ & $20 \%$ \\
\hline Predicted number of visitors & 4113872 & 3830151 & 494033 & 460529 & 486018 & 493752 \\
\hline Actual number of visitors & 4118754 & 4118754 & 536573 & 536573 & 495681 & 542639 \\
\hline
\end{tabular}

$\mathrm{O}=$ original coefficients.

$A=$ coefficients after removing autocorrelation.

where $g_{t}=$ year factor in year $t(t=0$ corresponds with $1970, t=1$ with 1971 , etc.) and $\min$ and $a$ are parameters. $g_{0}$ was set equal to one and using the estimated year factors for the years 1970 to 1976 as data, min and $a$ could be estimated ${ }^{3}$ and then the year factor for 1977 could be predicted. For an illustration of this year factor function, see Fig. 3.

The procedure was carried out for each season separately. The parameters min and $a$ were updated to predict the year factors for 1978 (using the visitor numbers over 1970-1977) and undated again to predict the year factors for 1979. The other parameters of the model were not updated however, so for the predictions for 1978 and 1979 the day, month, advertising coefficients, etc. estimated over 1970-1976 were used.

Table 2 shows that the model predicts very sat sfactory: the correlation coefficient between predicted and actual daily numbers of visitors is about 0.9 . There is some underprediction of the total number of visitors in a year (especially for 19\%7 and 1979), but since we want to use the mcdel to allocate advertising effort over the year, a goud prediction of the distribution of the visitors over the year is more important than a gcod

3 This was done by finding the least squares value (after a logarithmic transformation) of $a$ for each of a series of values for min and then taking the (min, a) combination with the smallest sum of squares. prediction of the general level of the number of visitors.

A notable result is that the quality of the prediction in Table 2 does not become worse as we get further away from the estimation period. So the 'underlying mechanism' generating the visitors seems to be rather stable. Therefore up to this point there is no reason to update the model by reestimating the parameters using the new data.

As indicated in Table 2 for the first two periods the prediction has also been carried out using the model coefficients obtained after the removal of autocorrelation. On the whole the predictions from the original coefficients are better, therefore these

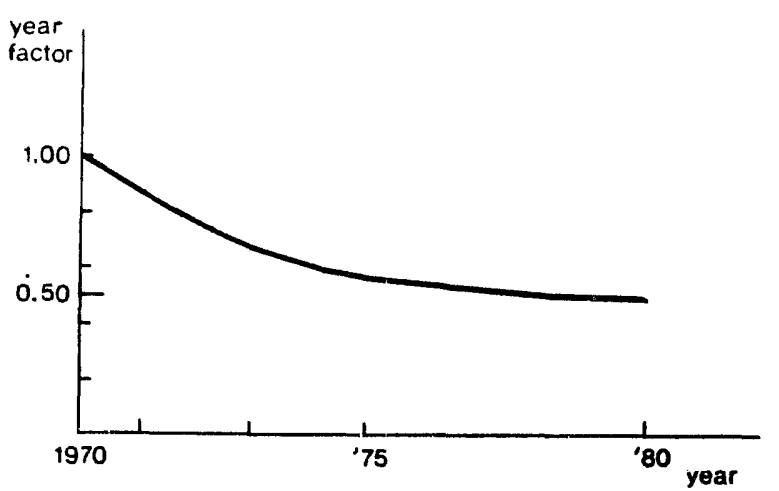

Fig. 3. Year factors for the summer season, based on the estimated eq. (9). 
coefficients are used in the optimization reported in the next section.

\section{Optimizing the advertising policy}

The model for the number of visitors, specified and parametrized in the previous sections was used to answer the questions: how much should be spent on advertising, which media should be used for advertising and when should the advertisements be placed? Although we speak of 'optimizing' the advertising policy here, no attempt was made to find the absolutely best policy. Because of the large number of influencing variables, this seemed too ambitious. Particularly it was tried to use dynamic programming. However, it turned out that the number of states (different budget levels, different intensity levels for TV, radio and newspaper advertising) was too large to make this technique practically feasible here. Therefore, a heuristic procedure was developed to use the information obtained in the previous sections to find better advertising schemes. The next subsection describes this procedure; subsequently the results obtained in this way are reported.

\subsection{The heuristic advertising planning procedure (HAP)}

The procedure basically is an incremental search procedure. The advertising budget is allocated on a step by step basis in such a way that for each additional budget slice advertisements are bought that maximally increase the number of visitors per additional guilder spent. Similar stepwise allocation procedures have been applied earlier in planning of promotional activity e.g. in media planning procedures such as the High Assay Model, see Moran [7] and in the CALLPLAN procedure developed by Lodish [6] Fig. 4.

The procedure is schematically depicted in

For a given year we take the period during which the park is open: March 1 to October 31, a period of 245 days. Given the total budget, the question is on which of these 245 days to place advertisements and which media to use. Of these 245 days the Sundays have to be excluded immediately since on Sunday there is no TV or radio advertising and there are no newspapers in the Netherlands.
For a specific year under study the dates of public holidays, school vacations, construction workers' vacation, etc. are known. For the weather variables the averages from the past are the best predictions. (In fact for each day we took the type of weather that during the last 18 years occurred mostly on the days with that serial number.) In this way for each of the $\mathbf{2 4 5}$ days a quantity can be computed that represents the number of visitors as determined by the non-advertising factor (the first part of (7)).

Here the year factor is dependent on the year for which the advertising planning is to be carried out and is determined by the negative growth curve (9).

After the non-advertising factors the effects of advertising in the previous years are taken into account by means of the last term of (7). Then the procedure starts assigning advertisements to days in the current year. One additional constraint was applied. Since the park has a maximum capacity (set at 14000 visitors a day) an advertisement is not placed if it would cause the number of visitors on one (or more) of the first 7 days after the placement to increase beyond that maximum capacity.

\subsection{Advertising schedules developed with HAP}

In using the heuristic advertising planning procedure we concentrate on the year 1977. For this year the optimal advertising schedule was developed with HAP compared with the actual schedule for 1977.

We took the year 1977 as basis for the comparison. However, the results for the allocation of the budget over media and over the various periods of the year can directly be generalized to other years. For a specific year one only has to account for the exact dates of the spring holidays (Easter, etc.) and the period of the construction workers' vacation which determines when the vacation peak falls. (In HAP this information can be given as input by the user and in this way HAP can be used for every year desired.)

In 1977 total advertising expenditures were Dfl 320000 distributed over the media as follows: $39 \%$ TV, $8 \%$ radio and 53\% daily newspaper advertising. Th; numbers of advertisements in the three media vere: 25,17 and 16 respectively. The tariffs per advertisement were: Dfl 5000 for TV, Dfl 1000 for radio and Dfl 10500 for newspapers (a combination of 15 ). 


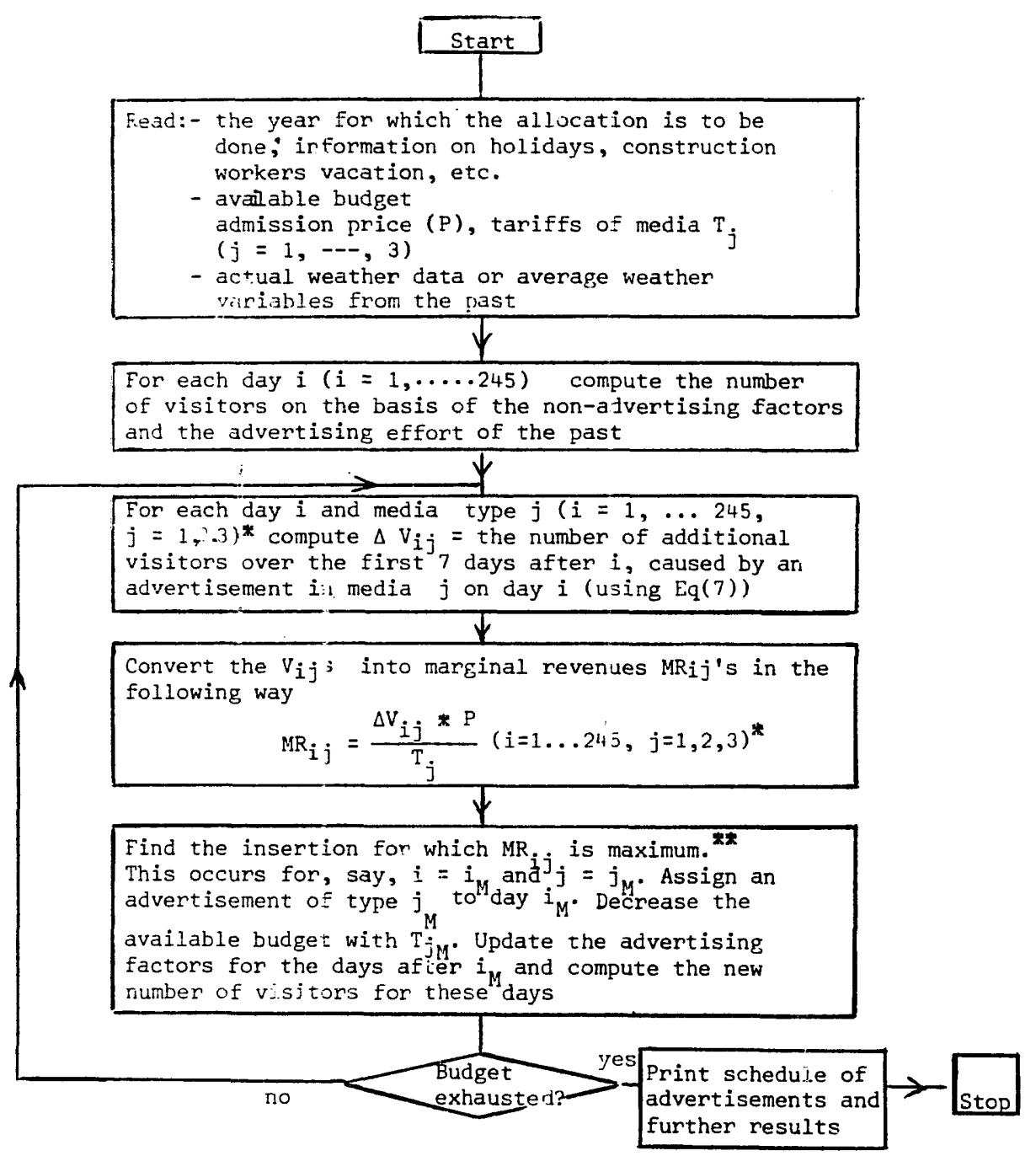

Fig. 4. Flow chart of heuristic advertising planning procedure (HAP).

* As far as applicable. For example according to the model no newspaper advertising is considered for the summer season (see Table 1).

** With the convention of no more than one advertisement of the same type on the same day.

We considered the situation where the same budget as was actually used in 1977, i.e. Dfl 320000 , was to be allocated. Table 3 gives the resulting schedule of advertisements, as compared with the actual schedule (indicated by stars).

Table 4 gives a summary of the actual advertising schedule (Case 1) and the schedule of Table 3 (Case 2 in Table 4) on a month-by-month basis.

Table 4 also shows that a better allocation of the budget increases the number of visitors from $485600^{4}$ to 595734 , i.e. by about 110000 visitors.

4485600 is the number of visitors predicted for 1977 for average weather conditions. The number 494038 in Table 2 is the total number of visitors predicted for 1977 given the actual weather conditions in that year.
With an average admission price of Dfl 4.75 , this means Dfl 523000 in additional revenues.

The optimal schedule in Table 3 differs considerably from the actual schedule. There is more TV and radio and much less emphasis on newspaper advertising in the optimal schedule. Moreover the distribution over the year is different in this schedule: a concentration of advertising around Easter, Ascensior Day and Whitsun, no advertising in March but more in September and, in general, a much less regular pattern of advertisements than in the actual schedule.

To examine the effects of introducing radio spots in spring/autumn, a response coefficient for radio advertising in spring/autumn was contrived 


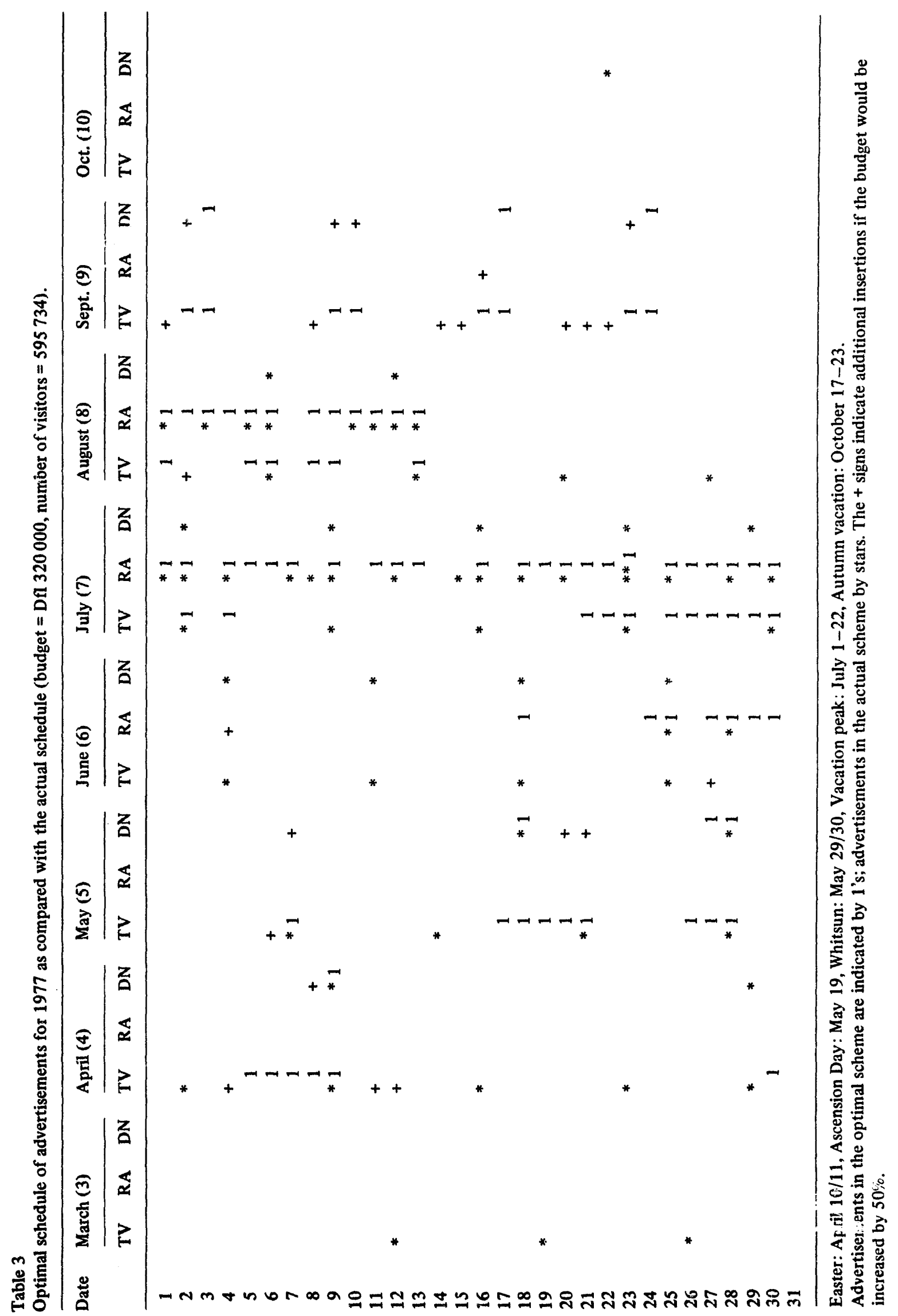


Table 4

Distribution of advertisements over the riedia and over the months in the actual schedule for 1977, as compared with a number of schedules, obtained for different conditions with HAP (numbers in brackets are number of visitors)

\begin{tabular}{|c|c|c|c|c|c|c|c|c|c|c|}
\hline & & March & April & May & June & July & Aug. & Sept. & Oct. & Total \\
\hline 1. Actual schedule & $\left\{\begin{array}{l}\text { TV } \\
\text { RA } \\
\text { DN }\end{array}\right.$ & $\begin{array}{l}3 \\
0 \\
0\end{array}$ & $\begin{array}{l}5 \\
0 \\
2\end{array}$ & $\begin{array}{l}4 \\
0 \\
2\end{array}$ & $\begin{array}{l}4 \\
3 \\
4\end{array}$ & $\begin{array}{r}5 \\
16 \\
5\end{array}$ & $\begin{array}{l}4 \\
8 \\
2 \\
(485\end{array}$ & $\begin{array}{l}0 \\
0 \\
0 \\
0\end{array}$ & $\begin{array}{l}0 \\
0 \\
1\end{array}$ & $\begin{array}{l}25 \\
27 \\
16\end{array}$ \\
\hline 2. Optimal allocation actual budget & $\left\{\begin{array}{l}\text { TV } \\
\text { RA } \\
\text { DN }\end{array}\right.$ & $\begin{array}{l}0 \\
0 \\
0\end{array}$ & $\begin{array}{l}6 \\
0 \\
1\end{array}$ & $\begin{array}{l}9 \\
0 \\
3\end{array}$ & $\begin{array}{l}0 \\
7 \\
0\end{array}$ & $\begin{array}{r}11 \\
23 \\
0\end{array}$ & $\begin{array}{c}6 \\
12 \\
0 \\
(5957\end{array}$ & $\begin{array}{l}8 \\
0 \\
3\end{array}$ & $\begin{array}{l}0 \\
0 \\
0\end{array}$ & $\begin{array}{r}40 \\
42 \\
7\end{array}$ \\
\hline $\begin{array}{l}\text { 3. Same stuation as } 2 \text {, but radio } \\
\text { advertising allowed } \\
\text { in spring/autumn season }\end{array}$ & $\left\{\begin{array}{l}\text { TV } \\
\text { RA } \\
\text { DN }\end{array}\right.$ & $\begin{array}{l}0 \\
0 \\
0\end{array}$ & $\begin{array}{r}7 \\
13 \\
2\end{array}$ & $\begin{array}{r}10 \\
18 \\
2\end{array}$ & $\begin{array}{l}0 \\
4 \\
0\end{array}$ & $\begin{array}{r}5 \\
22 \\
0\end{array}$ & $\begin{array}{l}0 \\
4 \\
0 \\
1679\end{array}$ & $\begin{array}{r}9 \\
19 \\
4 \\
\end{array}$ & $\begin{array}{l}0 \\
1 \\
0\end{array}$ & $\begin{array}{r}31 \\
81 \\
8\end{array}$ \\
\hline $\begin{array}{l}\text { 4. Same situation as } 2 \text {, additional } \\
\text { advertisements if budget } \\
\text { is increased by } 50 \%\end{array}$ & $\left\{\begin{array}{l}\text { TV } \\
\text { RA } \\
\text { DN }\end{array}\right.$ & $\begin{array}{l}0 \\
0 \\
0\end{array}$ & $\begin{array}{l}3 \\
0 \\
1\end{array}$ & $\begin{array}{l}1 \\
0 \\
3\end{array}$ & $\begin{array}{l}1 \\
1 \\
0\end{array}$ & $\begin{array}{l}0 \\
0 \\
0\end{array}$ & $\begin{array}{l}1 \\
0 \\
0 \\
1657\end{array}$ & $\begin{array}{l}7 \\
0 \\
5\end{array}$ & $\begin{array}{l}0 \\
0 \\
0\end{array}$ & $\begin{array}{r}13 \\
1 \\
9\end{array}$ \\
\hline
\end{tabular}

by assuming that the ratio between TV and radio response coefficients is the same in that season as in the summer season. As indicated in Table 4 (Case 3), radio advertising in spring/autumn seems very profitable.

\subsection{Varying the advertising budget}

HAP can also be used to study the effects of changes in the total advertising budget. Fig. 5 depicts the total number of visitors and the marginal number of visitors for budget levels ranging from 0 to Dfl 800000 , as computed by HAP. Thus for each budget level (in Fig. 5 the budget increases with steps of Dfl 100000) the allocation of the budget over media and in time is optimal in the sense of the HAP-procedure.

From Fig. 5 it can be observed that without any advertising in 1977 the total number of visitors as predicted by the model is 329611. Therefore roughly one third of the current number of visitors $(485600)$ would not have come if there had been

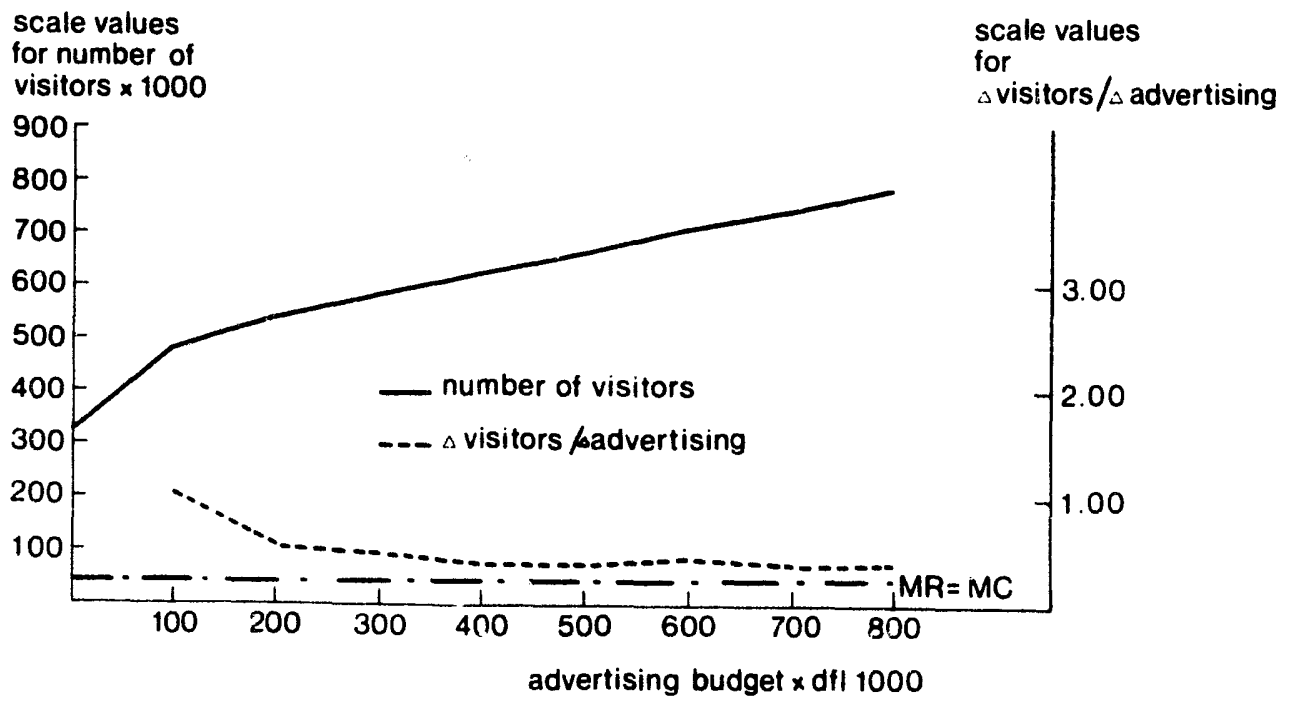

Fig. 5. Numbers of visitors and marginal number of visitors for different levels of the advertising budget. 
no advertising. It may be remarked that the order of magnitude of this drop in the number of visitors as predicted by the model (i.e. about 156000 ) is consistent with the actual decrease in the number of visitors in 1972, a year in which most of the advertising efforts was omitted ${ }^{5}$. This strengthens our belief in the validity of the model.

In Fig. 5 the level of the marginal number of visitors has been indicated that corresponds with the situation: $M R=M C$, i.e. where marginal revenues of advertising equal marginal costs.

This level is simply computed as one divided by admission price per visitor: $1 / 4.75=0.21$. Fig. 5 shows that for the whole budget interval considered the marginal number of visitors is about twice as high as this critical level, so that a budget increase would remain profitable even if the number of additional visitors were half the number predicted by the model. So at least there is no question of overspending on advertising. Some increase in the advertising budget seems advisable (of course provided that the budget is optimally allocated over media and in time). In Table 3 it has been indicated which additional advertisements are to be placed if the budget were increased by $50 \%$ (i.e. by Dfl 160000) (indicated by plus-signs). According to the model such a budget increase would generate 62000 additional visitors and increase the profit by about Dfl 135000 .

\subsection{Conclusions, recommendations and implementa- tion}

The results obtained with the heuristic advertising planning procedure HAP, as reported in the previous subsections lead to the following conclusions and recommendations.

(a) The effects of advertising on the number of visitors has clearly been demonstrated. It can be estimated that without advertising the park would lose about one third of its visitors.

(b) According to the model there are considerable advantages in readjusting the allocation of the current advertising budget with respect to media and time. The best schedule of advertisements found with the model generates about 110000 visitors more than the actual schedule. This best schedule is given in Table 3. The major adjustments are

5 As mentioned before the total drop in the number of visitors was about 200000 , which amounts to 160000 for the individual visitor to which our analysis refers.
(1) a different allocation of the budget over the media (with less emphasis on newspaper advertising and more on TV and radio),

(2) taking more advantage of major holidays and school vacations when placing the advertisements instead of making regular schedules of one spot per week and

(3) more advertisements in September instead of March.

Also the introduction of radio advertising in the spring/autumn season seems profitable.

(c) At the current level of advertising expenditure, additional revenues of increasing the budget are, according to the model, about twice as high as the costs. So anyhow there is no question of overspending on advertising. On the contrary, an increase in the advertising budget should seriously be considered. Additional advertising effort should be directed towards the months September, May and April in this order of priority.

\section{Implementation}

The first results of this study became available in February 1979. At that time it was only possible to make minor modifications in the advertising plan for 1979. On two aspects this schedule was changed into the direction of the recommendations: the TV-spots for March were omitted and two TV-spots were scheduled in September.

It was estimated after having observed th: actual numbers of visitors in March and September 1979 that this change increased the profit of the park with about Dfl 20000.

For the year 1980 the management of the park plans to follow the recommendation with respec: to the optimal advertising schedule more closely.

\section{Summary}

In this paper first a model was developed that predicts the daily number of visitors of a recreation park from the advertising efforts made by the park and a number of additional factors such as season, day, weather, etc. The model was parametrized using empirical data, gave a good fit for the estimation period and-more importantly predicted very well for subsequent periods.

The model was used in a heuristic allocation procedure that tries to allocate the advertising budget in an optimal way over the media: TV, radio and newspaper and over the days of the 
year. In this way it was possible to find advertising schedules that-for the same budge $\hat{\text { - }}$-attract considerably more visitors than the schedule currently used by the park. Using the allocation procedure it was also possible to study the effects of alternate levels of the advertising budget. The study resulted in several recommendations to the management of the park, a number of which have already been implemented.

\section{References}

[1] J. Johnston, Econometric Methods (McCraw-Hill, New York, 2nd ed., 1972).
[2] S.P. Ladany (Editor), Management Science Applications to Leisure Time Operations (North-Holland, Amsterdam, 1975).

[3] D.C. Little, BRANDAID: A marketing-mix model, Part 1: structure, Operations Res. 23 (1975) 628-55.

[4] D.C. Little, Aggregate advertising models: The state of the Art, Operations Res. 27(4) (1979) 629-667.

[5] D.C. Little and M. Lodish, A media planning calculus, Operations Res. 17 (1969) 1-35.

[6] M. Lodish, Callplan: An interactive salesman's call planning system, Management Sci. 18 (4, Part II) (1972) 25-40.

[7] W.T. Moran, Practical media decisions and the computer, J. Marketing (July 1963) 26-30.

[8] M.L. Vidale and H.B. Wolfe, An operations research study of sales response to advertising, Operations Res. 5 (1957) 370-381.

[9] C.B. Weinberg and M. Shachmut, Arts plan: A model based system for use in planning and performing arts series, Management Sci. 24 (1978) 654-64. 